\title{
Catastrophic catecholamine-induced cardiomyopathy rescued by extracorporeal membrane oxygenation in recurrent malignant pheochromocytoma
}

\author{
Daniel Min \\ Department of Cardiology, G SAM Hospital, Gunpo, Korea
}

Received: March 27, 2019

Revised: April 30, 2019

Accepted: May 8, 2019

Corresponding author:

Daniel Min

Department of Cardiology, G SAM

Hospital, 591, Gunpo-ro, Gunpo

15839, Korea

Tel: +82-31-389-3634

Fax: +82-31-389-3787

E-mail:danieruyo@gmail.com
Pheochromocytoma (PCC) is a rare catecholamine-producing tumor with the incidence in hypertension of $0.1-0.6 \%$. PCC crisis is an endocrine emergency that can lead to hemodynamic disturbance and organ failure such as catecholamine-induced cardiomyopathy. The circulatory collapse caused by it often requires mechanical support. The author reports an unusual case in which a patient who previously underwent surgery for malignant PCC developed catecholamine-induced cardiomyopathy, and successfully recovered using extracorporeal membrane oxygenation.

Keywords: Cardiogenic shock; Cardiomyopathy; Extracorporeal membrane oxygenation; Pheochromocytoma

\section{Introduction}

Pheochromocytoma (PCC) is a rare catecholamine-producing neuroendocrine tumor derived from chromaffin cells of the sympathetic nervous system. Because it can present with various clinical symptoms other than the classical triad of headache, palpitation and sweating, early diagnosis is not easy $[1,2]$. PCC crisis is a situation in which the secretion of catecholamine abruptly increases, caused by some spontaneous or specific stimuli. This is an endocrine emergency that can lead to hemodynamic disturbance and organ failure associated with significant mortality $[3,4]$. This situation often requires mechanical support, and some cases of effective recovery using extracorporeal membrane oxygenation (ECMO) are reported $[5,6]$. In a considerable number of cases, PCC is initially diagnosed due to the outbreak of a PCC crisis [3]. However, there are very few cases in which patients who have undergone surgical treatment for PCC are later diagnosed with a recurrence due to PCC crisis. Here, the author reports an unusual case in which a patient who previously underwent surgery for malignant PCC developed catecholamine induced cardiomyopathy after taking glucocorticoids, and successfully recovered using ECMO.

\section{Case}

A 44-year-old Asian female visited the emergency room complaining of a headache, nausea and vomiting. She had visited the hospital 11 years earlier for gestational hypertension. She had been diagnosed with PCC in the right adrenal gland (Fig. 1A) and successfully treated with a right adrenalectomy. PCC of the adrenal gland scaled score had been 7 , so the tumor had been diagnosed as malignant. For the first 5 years of follow-up, no signs of recurrence had been found, and she had not visited the hospital since then. Four hours before her visiting the present hospital, she took $4 \mathrm{mg}$ of methylprednisolone due to urticaria.

Her blood pressure (BP) was 180/100 $\mathrm{mmHg}$, and other vital 

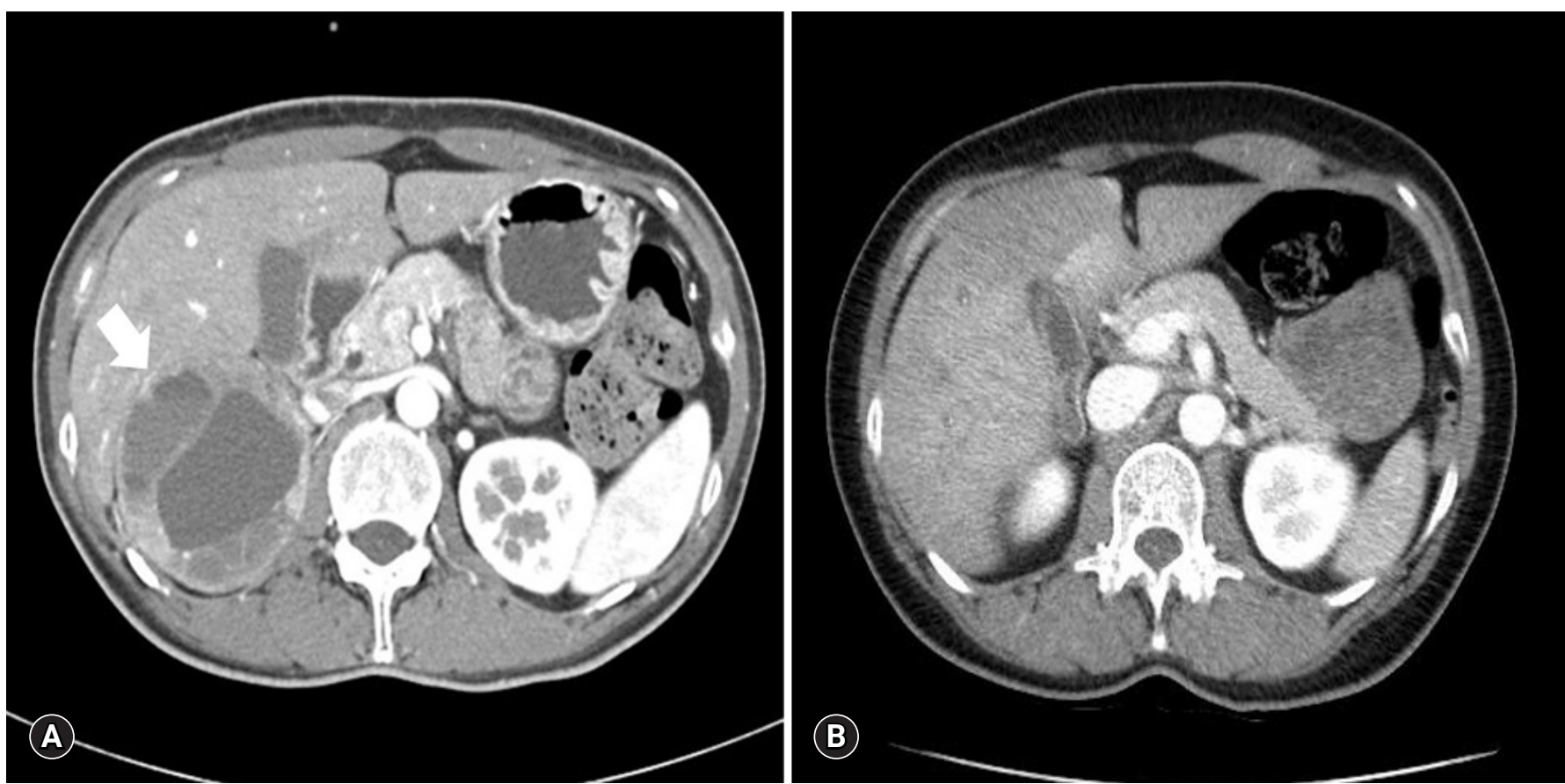

Fig. 1. (A) Abdomen-pelvis CT of 11 years ago shows a $9 \times 7 \mathrm{~cm}$ sized pheochromocytoma (arrow) in the right adrenal gland. (B) There is no evidence of local recurrence on abdomen-pelvis CT at admission. CT, computed tomography.

signs were stable. An electrocardiogram and a chest X-ray showed normal findings. She had no history of diabetes, but her glucose level was $330 \mathrm{mg} / \mathrm{dL}$, and bicarbonate level was reduced at 13.5 $\mathrm{mmol} / \mathrm{L}$. Other blood tests showed no abnormal findings, and urine dipstick analysis showed ketone $1+$. Hydration was performed with normal saline due to the possibility of early phase diabetic ketoacidosis. Abdomen-pelvis computed tomography (CT) was performed, and there was no evidence of local recurrence of PCC (Fig. 1B).

Four hours after visiting the hospital, she complained of severe dyspnea. She showed tachycardia with a pulse of 130 beats/min, severe tachypnea with a respiratory rate of over 35 breaths/min, and a fever of $38^{\circ} \mathrm{C}$. Rale was heard from both lung fields, and a chest X-ray revealed a rapidly developed bilateral consolidation (Fig. 2). Intubation and mechanical ventilation were performed because of severe hypoxemia. According to echocardiography conducted at bedside, although the cardiac chamber size was normal, there was severe global hypokinesia of the left and right ventricles. The left ventricular ejection fraction was approximately $20 \%$ and the tricuspid annular peak velocity had decreased to $6 \mathrm{~cm} / \mathrm{s}$, indicating severe ventricular dysfunction of both ventricles. Antibiotics and diuretics were used, considering the possibility of acute respiratory distress syndrome due to atypical pneumonia or acute pulmonary edema caused by heart failure. She was moved to the intensive care unit from the emergency
Table 1. Plasma levels of catecholamines

\begin{tabular}{lrc}
\hline Catecholamine & \multicolumn{1}{c}{ Level } & Normal range $\left(\times 10^{8} / \mathrm{kg}\right)$ \\
\hline Epinephrine & $19,752.5$ & $0-140 \mathrm{pg} / \mathrm{mL}$ \\
Norepinephrine & $5,641.0$ & $70-1,700 \mathrm{pg} / \mathrm{mL}$ \\
Metanephrine & 46.1 & $0-0.5 \mathrm{nmol} / \mathrm{L}$ \\
Normetanephrine & 23.5 & $0-0.9 \mathrm{nmol} / \mathrm{L}$
\end{tabular}

room. Five hours after intubation, her BP was dropped to $60 / 30$ $\mathrm{mmHg}$ and pulse gradually slowed. One $\mathrm{mg}$ of epinephrine and $1 \mathrm{mg}$ of atropine were injected immediately, but asystole occurred. Cardiopulmonary resuscitation was performed immediately and venoarterial ECMO was prepared. ECMO was initiated 15 minutes after arrest. Asystole and return of circulation were repeated 3 times for 42 minutes. After that, the patient's rhythm was restored and no further cardiac arrest occurred. She was treated for 5 days with support of ECMO, mechanical ventilation, and inotropic agents. She recovered consciousness and ECMO was removed when her vital signs became stable without inotropics. After 10 days, ventilator weaning and extubation were performed.

The results of hormone tests performed at the time of hospitalization showed an overall increase in catecholamine (Table 1), and catecholamine-induced cardiomyopathy due to PCC was strongly suspected. In order to confirm the recurrence of PCC, a ${ }^{131}$ I-metaiodobenzylguanidine (MIBG) scan was performed, but 

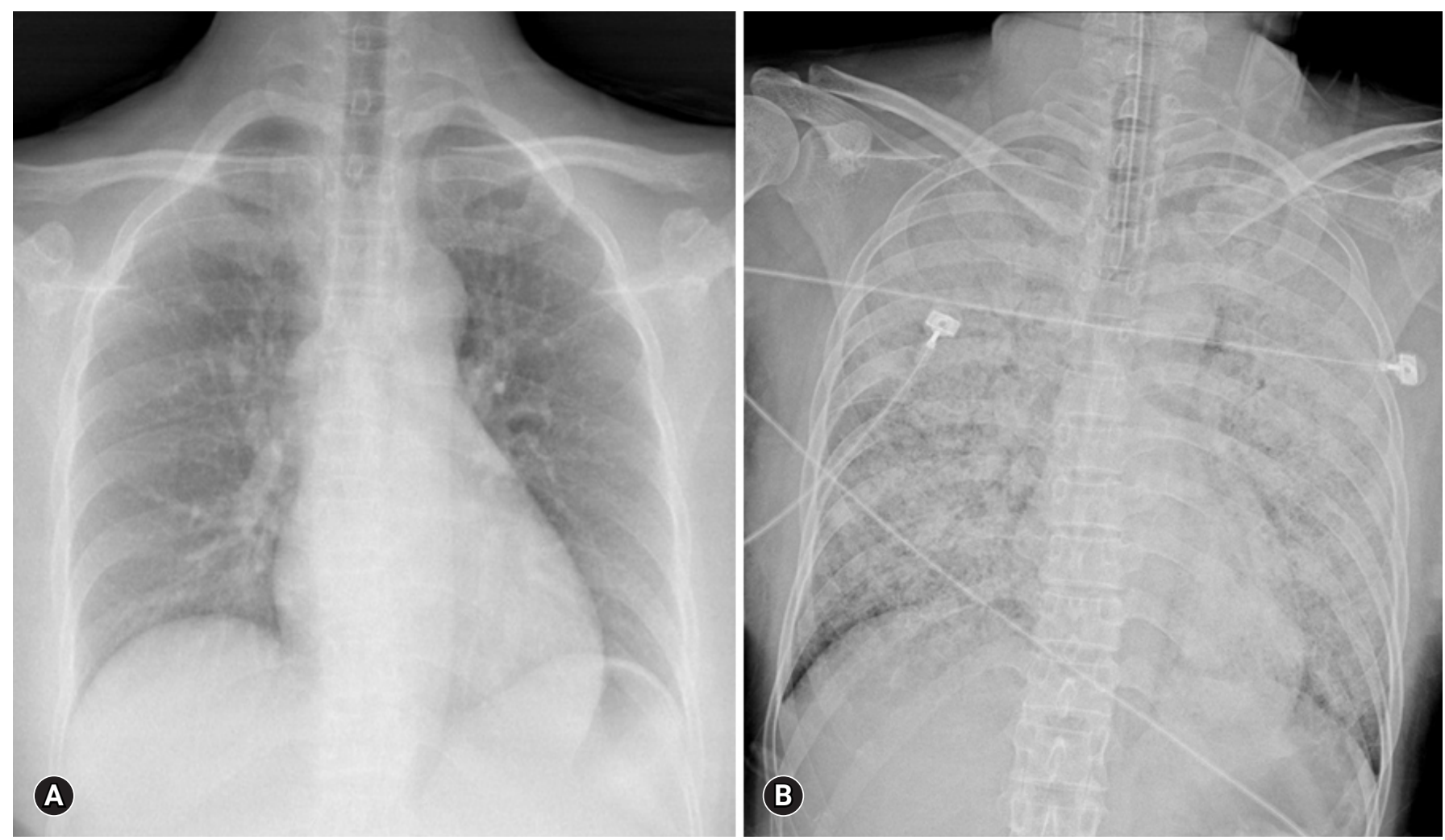

Fig. 2. (A) No abnormality is seen on chest X-ray at admission. (B) Chest X-ray after 4 hours of admission reveals a rapidly developed bilateral consolidation.

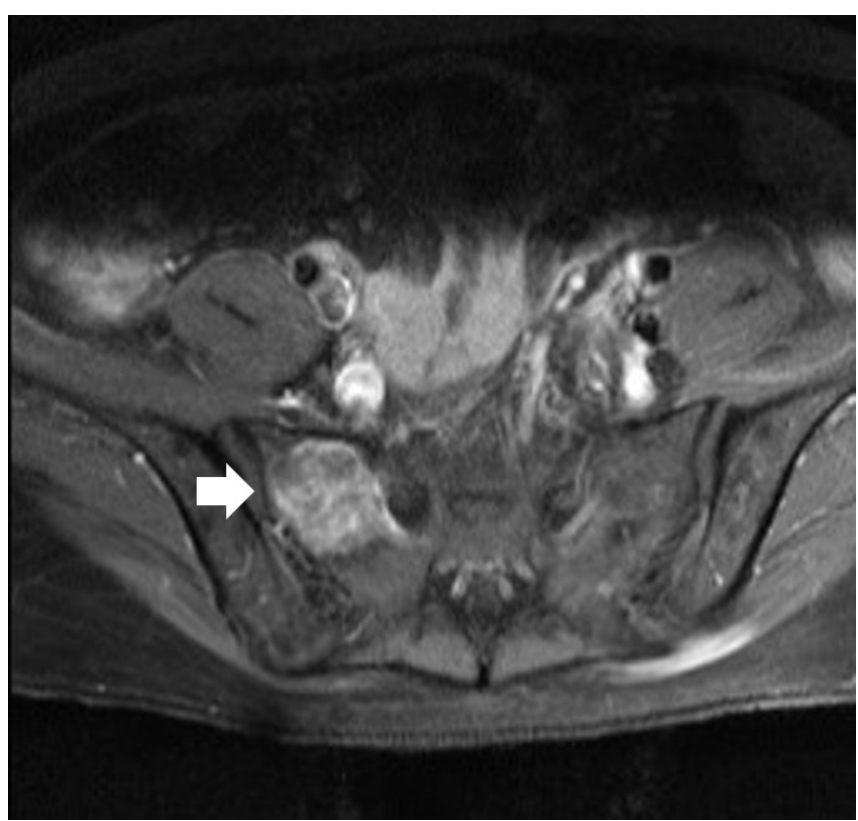

Fig. 3. Sacrum magnetic resonance imaging shows a $2.7 \times 2.9 \times 2.8$ $\mathrm{cm}$ sized well-defined, lobulated, and slightly expansile mass (arrow) at right upper sacral ala. no focal radioactive uptake lesion was shown. The patient's CT was reviewed closely, and a faint mass-like lesion was detected on her right sacrum. Sacrum magnetic resonance imaging was performed, and a $2.7 \times 2.9 \times 2.8 \mathrm{~cm}$ sized well-defined, lobulated, and slightly expansile mass with mainly $\mathrm{T} 1$ low T2 high signal intensity and heterogeneous enhancement at right upper sacral ala was seen (Fig. 3). Other small lesions, which are assumed to be metastasis, were also found at the $\mathrm{S} 2$ level of the sacrum, right iliac bone, and lower L4 vertebral body. A positron emission tomography scan showed an increase of uptake in the same regions (Fig. 4). For a histological verification, an open bone biopsy was performed. Atypical spindle-shaped tumor cells showing synaptophysin and chromogranin positivity were found upon histopathologic examination (Fig. 5). These results demonstrated that the lesions on the sacrum were recurrent metastatic PCC.

In the patient's follow-up, the cardiac functions, including left and right ventricles, were found to be completely recovered, with $65 \%$ of ejection fraction. Coronary angiography performed after ventilator weaning showed no abnormal findings. Currently, her $\mathrm{BP}$ is being successfully regulated with an $\alpha$-blocker (doxazocin) and $\beta$-blocker (carvedilol) and treatment for the recurrent PCC is planned. 


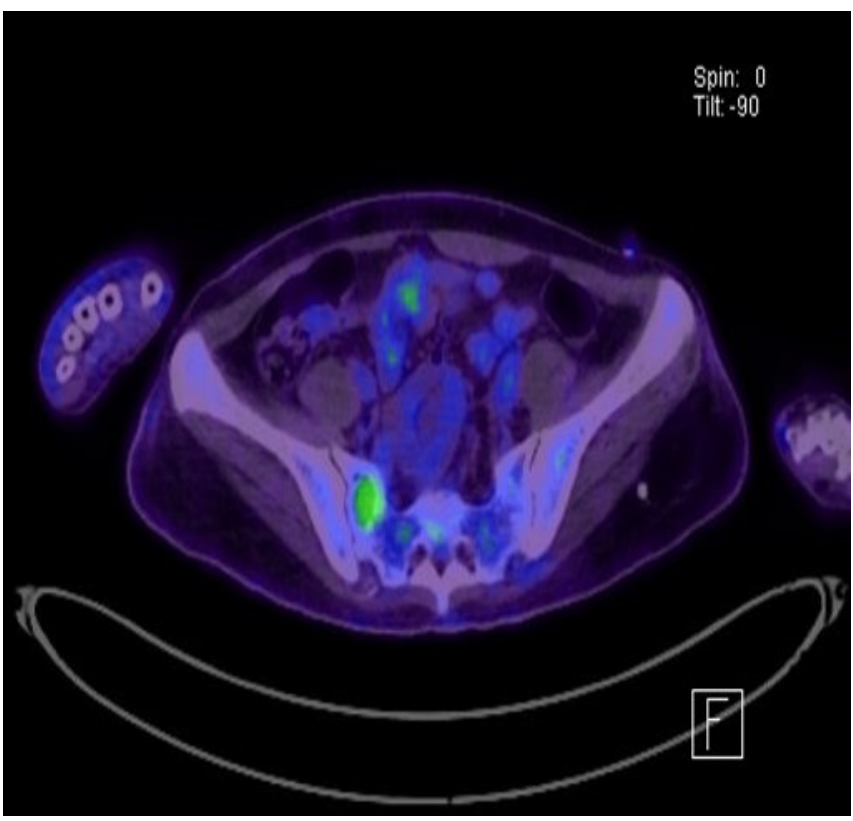

Fig. 4. Positron emission tomography scan shows an increase of uptake in the mass of sacrum.

\section{Discussion}

Classic symptoms of PCC are headache, palpitation and sweating. However, many patients show atypical symptoms and it is not difficult to find cases with PCC crisis as the first symptom $[1,2]$. PCC crisis involves suddenly released cathecholamines that act on $\alpha$-adrenergic receptors to cause arterial contraction, along with reduction in intravascular effective volume, which ultimately causes cellular ischemia to have a systemic effect [3]. It often causes cardiovascular emergencies, such as myocardial infarction, catecholamine-induced cardiomyopathy, and acute myocarditis, which eventually lead to progression of circulatory collapse. The mechanism of cardiac injury includes direct cardiac myocyte damage of catecholamine, increased myocardial oxygen demand and decreased oxygen supply due to coronary artery spasm [4].

The key to treat PCC crisis is to recognize early and stabilize the patient's vital sign until the causes are corrected, but medical treatment alone is not enough in some cases. There have been reports of using mechanical support, such as intraaortic balloon pump or ECMO, in effectively treating such cases $[5,6]$. In this case, the patient was effectively rescued with timely use of ECMO even though the patient had suffered a cardiac arrest. Fortunately, most catecholamine induced cardiac injuries are reversible, and as such, many cases including this case show recovery of damaged cardiac functions later on [7]. Therefore, if necessary, more ag- gressive mechanical support should be considered during the unstable period of PCC crisis. It can save the patients from the vicious cycle and increases their chances for cardiac function recovery, which would ultimately increase the survival rate [8].

Causes of PCC crisis include trauma, surgery, and anesthesia, as well as drugs such as glucagon, radiocontrast agent, tyramine, tricyclic antidepressant, and metoclopramide [3]. Cases occurring after glucocorticoid administration are very rare, with only about 20 cases reported to date [9-11]. In this case, methylprednisolone taken prior to admission was suspicious of the cause of PCC crisis. Although it was a low dose, no other possible causes were found, and since PCC crisis also occurred in another case when $2 \mathrm{mg}$ of dexamethasone was administered during dexamethasone suppression test [9], the possibility of $4 \mathrm{mg}$ dose of methylprednisolone being the cause was believed to be sufficient enough.

Two possible mechanisms by which glucocorticoid causes PCC crisis can be considered. First, glucocorticoid promotes the production and release of catecholamines. In normal adrenal gland, the adrenal medulla is already exposed to excessive amount of glucocorticoid secreted from nearby adrenal cortex, and thus, it is not significantly affected by introduction of external glucocorticoid. However, PCC is not surrounded by the adrenal cortex and has a modified vascular structure, and as a result, it has lost the ability to regulate external steroids and overreacts to them [12]. Glucocorticoid activates enzymes involved in catecholamine metabolism, production, and secretion in PCC, thereby promoting catecholamine secretion [13]. Second, glucocorticoid reinforces the actions of catecholamines in the peripheral vascular tissues by suppressing the formation of endothelial-derived vasodilators such as nitric oxide and prostacyclin [14].

The point of interest in this case is that PCC crisis occurred in a patient who was already being treated for malignant PCC and recurrence was confirmed through such event, as well as this crisis was induced by steroids. PCC itself is not a common disease, and recurrences are very rare with only $4.6-6.5 \%$ [15]. The prognosis of malignant PCC is poor and the 5-year survival rate is reported to be $20-50 \%$ [16]. Poor prognosis is associated with local recurrence or distant metastasis, which often appear within the first 2 years from the initial diagnosis, but such occurrence even after 40 years has also been reported [17]. In this case, bone metastasis might had proceeded 5 years after surgery. In the meantime, catecholamine was not being secreted in excessive amounts to cause symptoms, but catecholamine secretion did increase suddenly when steroids were taken, which is believed to have caused the PCC crisis.

In this case, we could not easily find the lesion, because there 


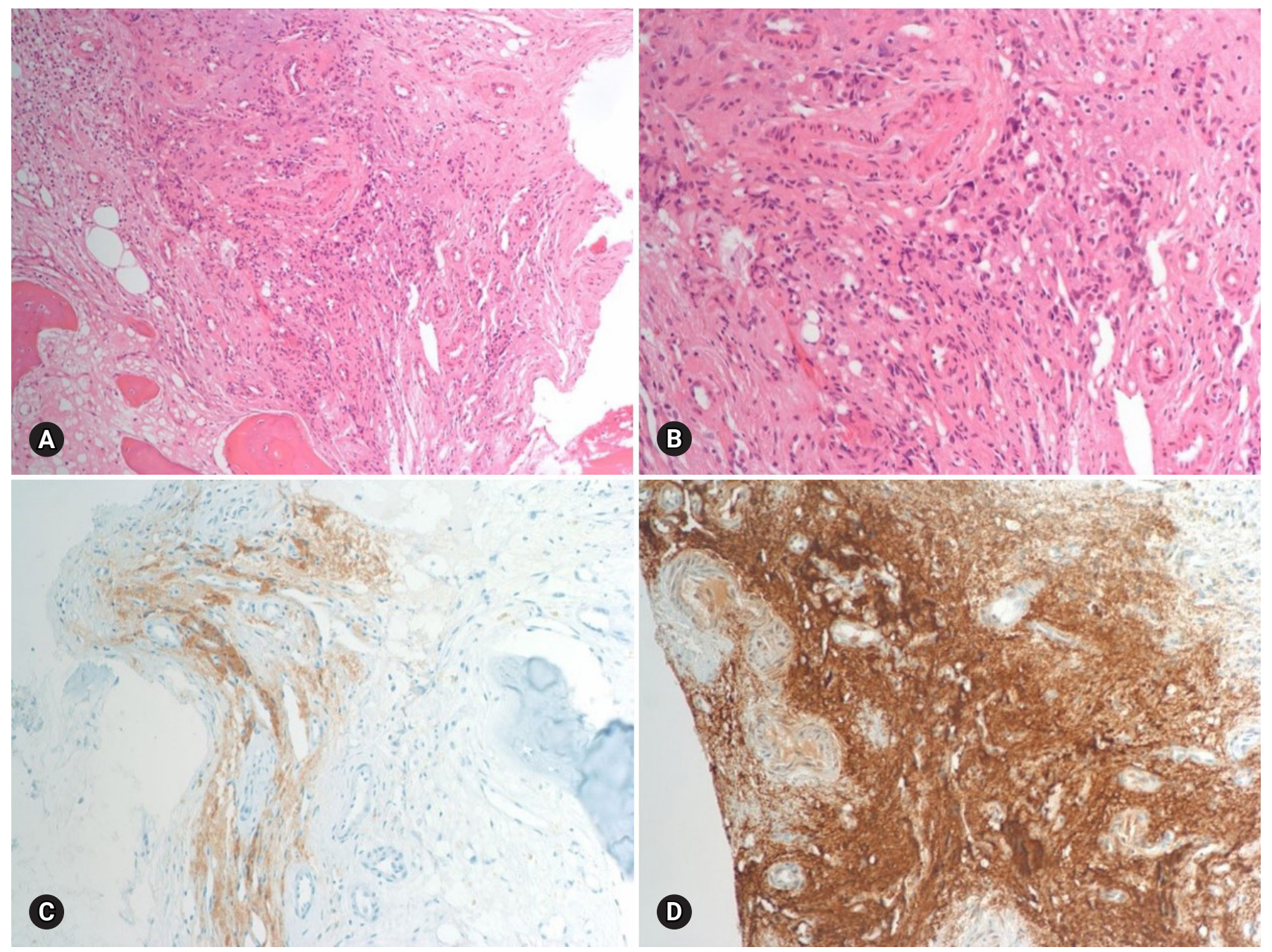

Fig. 5. Histopathologic findings of the sacral mass. There are atypical spindle-shaped tumor cells (hematoxylin and eosin stain, $\times 100[A]$ and $\times 200[B])$ show synaptophysin (C) and chromogranin (D) positivity $(\times 100)$.

was no focal recurrence on $\mathrm{CT}$ and no focal radioactive uptake lesion showing on the ${ }^{131}$ I-MIBG scan. The MIBG scan is an effective functional imaging procedure for detecting PCC. Its specificity is quite high (95-100\%), but the sensitivity is relatively low (approximately 77-90\%). The false-negative of the MIBG scan can be affected by tumor size, drugs that interfere with iodine uptake, amount of iodine isotope used, imaging time etc. Several studies have reported that the sensitivity of the MIBG scan is higher for adrenal PCC than for extra-adrenal PCC indicating that tumor location also affects sensitivity [18]. In this case, because the recurrent lesion was a sacrum, extra-adrenal lesion, it would not have been found with an MIBG scan.

In conclusion, PCC crisis is a very serious condition that can often be life threatening, and as such, the patient needs to be treated carefully; and if necessary, mechanical support should be considered without hesitation. In addition, it can be caused by various medications, and since steroids that are commonly prescribed can also be a cause, although rare, caution should be taken when prescribing steroids. Attention should be paid not only to patients that are currently suspected of having PCC, but also patients with prior treatment history as well.

\section{Conflicts of interest}

No potential conflicts of interest relevant to this article was reported.

\section{ORCID}

Daniel Min, https://orcid.org/0000-0003-0573-6351 


\section{References}

1. Lenders JW, Eisenhofer G, Mannelli M, Pacak K. Phaeochromocytoma. Lancet 2005;366:665-75.

2. Lenders JW, Duh QY, Eisenhofer G, Gimenez-Roqueplo AP, Grebe SK, Murad MH, et al. Pheochromocytoma and paraganglioma: an endocrine society clinical practice guideline. J Clin Endocrinol Metab 2014;99:1915-42.

3. Whitelaw BC, Prague JK, Mustafa OG, Schulte KM, Hopkins PA, Gilbert JA, et al. Phaeochromocytoma crisis. Clin Endocrinol (Oxf) 2014;80:13-22.

4. Prejbisz A, Lenders JW, Eisenhofer G, Januszewicz A. Cardiovascular manifestations of phaeochromocytoma. J Hypertens 2011;29:2049-60.

5. Suh IW, Lee CW, Kim YH, Hong MK, Lee JW, Kim JJ, et al. Catastrophic catecholamine-induced cardiomyopathy mimicking acute myocardial infarction, rescued by extracorporeal membrane oxygenation (ECMO) in pheochromocytoma. J Korean Med Sci 2008;23:350-4.

6. Sojod G, Diana M, Wall J, D’Agostino J, Mutter D, Marescaux J. Successful extracorporeal membrane oxygenation treatment for pheochromocytoma-induced acute cardiac failure. Am J Emerg Med 2012;30:1017.e1-3.

7. Giavarini A, Chedid A, Bobrie G, Plouin PF, Hagège A, Amar L. Acute catecholamine cardiomyopathy in patients with phaeochromocytoma or functional paraganglioma. Heart 2013;99:1438-44.

8. Otusanya O, Goraya H, Iyer P, Landi K, Tibb A, Msaouel P. A vicious cycle of acute catecholamine cardiomyopathy and circulatory collapse secondary to pheochromocytoma. Oxf Med Case Reports 2015;2015:343-5.

9. Yi DW, Kim SY, Shin DH, Kang YH, Son SM. Pheochromocyto- ma crisis after a dexamethasone suppression test for adrenal incidentaloma. Endocrine 2010;37:213-9.

10. An JY, Kim DR, Oh JY, Han YC, Lee IS, Kown TJ, et al. A case of recurrent glucocorticoid-induced pheochromocytoma crisis during the treatment of urticarial. Korean J Med 2015;88:564-9.

11. Barrett C, van Uum SH, Lenders JW. Risk of catecholaminergic crisis following glucocorticoid administration in patients with an adrenal mass: a literature review. Clin Endocrinol (Oxf) 2015;83:622-8.

12. Tischler AS, Perlman RL, Morse GM, Sheard BE. Glucocorticoids increase catecholamine synthesis and storage in PC12 pheochromocytoma cell cultures. J Neurochem 1983;40:36470.

13. McMahon A, Sabban EL. Regulation of expression of dopamine beta-hydroxylase in PC12 cells by glucocorticoids and cyclic AMP analogues. J Neurochem 1992;59:2040-7.

14. Yang S, Zhang L. Glucocorticoids and vascular reactivity. Curr Vasc Pharmacol 2004;2:1-12.

15. Harari A, Inabnet WB 3rd. Malignant pheochromocytoma: a review. Am J Surg 2011;201:700-8.

16. Baudin E, Habra MA, Deschamps F, Cote G, Dumont F, Cabanillas $\mathrm{M}$, et al. Therapy of endocrine disease: treatment of malignant pheochromocytoma and paraganglioma. Eur J Endocrinol 2014;171:R111-22.

17. Tanaka S, Ito T, Tomoda J, Higashi T, Yamada G, Tsuji T. Malignant pheochromocytoma with hepatic metastasis diagnosed 20 years after resection of the primary adrenal lesion. Intern Med 1993;32:789-94.

18. Ilias I, Divgi C, Pacak K. Current role of metaiodobenzylguanidine in the diagnosis of pheochromocytoma and medullary thyroid cancer. Semin Nucl Med 2011;41:364-8. 